\title{
Cooperación en ciencia y tecnología entre Argentina y Cuba en el siglo XXI. El caso del Centro Argentino-Cubano de Biotecnología Aplicada al Desarrollo de Vacunas y Fármacos (2009-2015)*
}

\author{
Scientific and technological cooperation between Argentina and Cuba in the $21^{\text {st }}$ \\ century. The case of the Argentine-Cuban Center for Biotechnology Applied to the \\ Development of Vaccines and Drugs (2009-2015)
}

\begin{abstract}
María Paz López ${ }^{* *}$
Centro de Estudios Interdisciplinarios en Problemáticas Internacionales y Locales (CEIPIL)

Universidad Nacional del Centro de la Provincia de Buenos Aires (UNICEN)

Comisión de Investigaciones Científicas de la Provincia de Buenos Aires (CIC)

Argentina.
\end{abstract}

Cómo citar este artículo: López, M. P. (2019). Cooperación en ciencia y tecnología entre Argentina y Cuba en el siglo XXI. El caso del Centro Argentino-Cubano de Biotecnología Aplicada al Desarrollo de Vacunas y Fármacos (2009-2015). Si Somos Americanos, 19(1),

pp.139-164.

DOI: $10.4067 / \mathrm{S} 0719-09482019000100139$

\section{Resumen}

El presente trabajo examina la cooperación científico-tecnológica entre Argentina y Cuba en el período 2009-2015, tomando como caso de estudio el Centro Argentino-Cubano de

Esta indagación se realizó en el marco de una investigación más amplia, correspondiente a la instancia de una beca posdoctoral otorgada por el Consejo Nacional de Investigaciones Científicas y Técnicas de Argentina. A su vez, se inserta en el marco del proyecto "Inserción internacional y política en ciencia, tecnología e innovación: dinámicas, instrumentos y actores en la Argentina (2007-2015)”, acreditado por la Secretaría de Políticas Universitarias del Ministerio de Educación (Código 03/D285).

** Profesora en Ciencias de la Educación, Universidad Nacional del Centro de la Provincia de Buenos Aires, Argentina; magíster en Ciencia, Tecnología y Sociedad, Universidad Nacional de Quilmes, Argentina; doctora en Ciencias Sociales, Universidad Nacional de La Plata, Argentina. Centro de Estudios Interdisciplinarios en Problemáticas Internacionales y Locales (CEIPIL), Universidad Nacional del Centro de la Provincia de Buenos Aires (UNICEN), Comisión de Investigaciones Científicas de la Provincia de Buenos Aires (CIC). Docente en la Facultad de Derecho y Facultad de Ciencias Humanas, UNICEN. Correo electrónico: mpaz_lo@yahoo.com.ar 
Biotecnología Aplicada al desarrollo de Vacunas y Fármacos, desarrollado conjuntamente por el Ministerio de Ciencia, Tecnología e Innovación Productiva argentino y contrapartes cubanas. Desde el punto de vista teórico, recupera aportes de la sociología de la ciencia, las relaciones internacionales y la política científica para el abordaje de las dinámicas microsociales de la cooperación internacional, así como la incidencia de la política exterior y de la política científico-tecnológica en dicho proceso. Se concluye que la política científico-tecnológica argentina del período, en consonancia con los lineamientos de política exterior, otorgó importancia a la cooperación con países latinoamericanos, en particular con naciones caribeñas, entre las que se destaca Cuba. En este contexto, dotó de un marco formal y un financiamiento específico a lazos argentino-cubanos preexistentes para forjar un desarrollo más estable y dinámico de los mismos, no sin encontrar ciertas limitaciones.

Palabras clave: cooperación internacional, ciencia y tecnología, biotecnología.

\begin{abstract}
This article examines scientific-technological cooperation between Argentina and Cuba between 2009 and 2015, taking as a case study the Argentine-Cuban Center of Biotechnology Applied to the Development of Vaccines and Drugs, which was jointly developed by the Ministry of Science, Technology and Productive Innovation of Argentina and its Cuban counterparts. From the theoretical perspective, the article engages with the contributions of the Sociology of Science, International Relations and Scientific Policy to address the micro-social dynamics of international cooperation, as well as the impact of foreign policy and scientific-technological policy regarding this process. The author concludes that Argentina's scientific-technological policy and foreign policy guidelines emphasized cooperation with Latin American countries and particularly Caribbean nations, notably Cuba. Within this framework, it provided a formal framework and special funding for existing Argentine-Cuban ties and for a more stable and dynamic development of the same, albeit encountering certain limitations.
\end{abstract}

Keywords: international cooperation, science and technology, biotechnology

\title{
Introducción
}

El objetivo general del artículo es analizar la cooperación científico-tecnológica entre Argentina y Cuba, tomando como caso de estudio el Centro Argentino-Cubano en Biotecnología Aplicada al Desarrollo de Vacunas y Fármacos, en el período 2009-2015. Se considera relevante avanzar en tal sentido, en tanto se observa una vacancia de aportes 
relativos al análisis de la cooperación entre Argentina y Cuba en materia de ciencia y tecnología. Si bien existen trabajos que caracterizan los sistemas científico-tecnológicos cubanos (Núñez Jover, 2005) y argentino (Albornoz y Gordon, 2011), así como textos referentes a la relación argentino-cubana en el marco de la política exterior del país (Aranda, 2004; Colombo y Giglio, 2011), la cuestión de la cooperación en ciencia y tecnología entre ambas partes no parece haber sido objeto de análisis.

Por su parte, los trabajos abocados al estudio de la cooperación latinoamericana en ciencia y tecnología se han centrado en organismos regionales sudamericanos como el Mercado Común del Sur (MERCOSUR) (Marí, Estébanez y Suárez, 2001; Velho, 2000), quedando así excluido del análisis el caso cubano. Como excepción, un estudio desarrollado por Kern (2014) avanza en la identificación de la agenda científico-tecnológica en el caso del MERCOSUR, la Unión de Naciones Suramericanas (UNASUR) y la Comunidad de Estados Latinoamericanos y Caribeños (CELAC). Este último organismo cuenta con la presencia de ambos países entre sus miembros.

Desde el punto de vista de la publicación conjunta, un análisis de la colaboración científica entre los países de América Latina a través de documentos registrados entre 1975-2004 en el Science Citation Index versión Web of Science, advierte que la colaboración bilateral entre Brasil y Argentina ha dominado, sobre todo a partir de la creación del MERCOSUR en 1991, y que en la última década se advierte una presencia importante de trabajos bilaterales entre México y Cuba. Asimismo, el estudio señala una tendencia de menores niveles de colaboración con Argentina y Brasil para los países más alejados geográficamente, entre los que se encuentra Cuba (Russell, Ainsworth, Del Río, NarváezBerthelemot y Cortés, 2007). Por su parte, es preciso contemplar el carácter informal de muchos de los vínculos internacionales desarrollados por los propios investigadores. Sobre estos vínculos, no se han encontrado trabajos.

En este marco, el presente trabajo analiza la cooperación científico-tecnológica entre Argentina y Cuba durante el período señalado, recuperando aportes de la sociología de la ciencia, las relaciones internacionales y la política científica para la comprensión de las dinámicas microsociales de la cooperación y la contextualización de las mismas en el marco de la política exterior y la política científico-tecnológica de los países. Específicamente, se centra en el caso del Centro Argentino-Cubano de Biotecnología Aplicada al desarrollo de Vacunas y Fármacos (en adelante CACBVaF), creado conjuntamente por el Ministerio de Ciencia, Tecnología e Innovación Productiva (en adelante MINCYT) y contrapartes cubanas en 2009. 


\section{Decisiones teórico-metodológicas para el abordaje del CACBVaF}

La cooperación científico-tecnológica internacional es entendida como una asociación dada en el ámbito de la investigación y la innovación entre actores de distintos países, en la que los objetivos son acordados conjuntamente y los beneficios resultan mutuos (Sebastián y Benavides, 2007). Esta definición destaca la importancia de la complementariedad de las capacidades, así como de la presencia de cierta simetría, confianza y reconocimiento mutuo como motor de la cooperación. El establecimiento de este tipo de cooperación implica una valoración de las capacidades de las contrapartes - los desarrollos científicos y tecnológicos en determinadas áreas-, donde cada una aporta conocimientos, recursos humanos calificados, infraestructura, insumos, entre otros. A su vez, la cooperación se construye en torno a identidades e intereses políticos y económicos, siendo atravesada por desigualdades económicas y políticas internacionales (Kern, 2009).

El presente trabajo comprende la cooperación internacional en ciencia y tecnología como un fenómeno atravesado por diferentes dinámicas: tanto las propias prácticas internacionales de los investigadores, como los lineamientos de política exterior y de política científico-tecnológica. La perspectiva de los 'estudios de laboratorio' de la sociología de la ciencia ha permitido abrir la 'caja negra' de los programas, centros y laboratorios de investigación; desarrollar una visión más próxima al fenómeno bajo estudio; dar voz a los propios científicos y prestar atención a las decisiones, criterios y actividades que desarrollan en la vida cotidiana (Kreimer, 1999). Por su parte, la política exterior de un país -objeto de estudio de la disciplina de las Relaciones Internacionales- posibilita la identificación del catálogo de países o regiones con quienes se promueve la cooperación científico-tecnológica (Kern, 2014).

A su turno, la política científico-tecnológica - analizada desde la política científica como área de conocimiento- permite comprender las capacidades de investigación, desarrollo e innovación a partir de las cuales el país se proyecta internacionalmente en la materia, atrae socios en el exterior y desarrolla actividades de cooperación internacional. Asimismo, posibilita la identificación de los objetivos políticos, sociales y económicos más amplios que guían el establecimiento de agendas de interés, temáticas estratégicas y áreas de vacancia hacia las cuales se encauzan los esfuerzos científico-tecnológicos nacionales y las actividades de cooperación internacional (Herrera, 2015; Hurtado, 2012).

Bajo esta mirada multidimensional, el artículo se centra en el caso del Centro ArgentinoCubano de Biotecnología aplicada al desarrollo de Vacunas y Fármacos (CACBVaF), una iniciativa de cooperación binacional promovida desde la esfera gubernamental en un área central para el desarrollo de la economía y la salud, como es la biotecnología. El recorte temporal del estudio se extiende desde 2009, año en que se crea el Centro a partir de un Acuerdo Marco firmado por ambas naciones, hasta 2015, momento en que se identifica la finalización de los proyectos conjuntos desarrollados en su seno. Las fuentes de 
información utilizadas en este artículo han sido secundarias (documentos emitidos por el MINCYT y el Ministerio de Relaciones Exteriores y Culto, MREC) y primarias (entrevistas realizadas a directores argentinos de proyectos seleccionados y financiados en el marco del Centro). El análisis se apoyó sobre artículos científicos relativos a los distintos tópicos abordados.

Específicamente, se utilizaron los documentos oficiales consignados a continuación: "Acuerdo Marco para la creación del Centro Binacional Argentino-Cubano de Biotecnología aplicada al Desarrollo de Vacunas y Fármacos" y listado de "Proyectos en ejecución", disponibles en la página web del MINCYT; y "Lineamientos para la cooperación sur-sur (2013-2015)", publicado por el MREC. También se trabajó con comunicados de prensa del MINCYT. ${ }^{1} \mathrm{El}$ análisis cualitativo de estos documentos procuró describir internamente su contenido en términos de contrapartes involucradas, fecha de creación, relevancia temática, objetivos, modalidades, organización institucional y financiamiento. Asimismo, se desarrolló un análisis externo, buscando comprender el documento en el conjunto de circunstancias sociales, políticas y científico-tecnológicas en que surgió (López Noguero, 2002).

La recolección de datos también se realizó a través de entrevistas semiestructuradas mantenidas con los directores argentinos de proyectos seleccionados y financiados por el CACBVaF. El listado de los cinco proyectos activos en el marco del Centro, disponible en la página web del MINCYT, facilitó el proceso de identificación de los entrevistados, al mencionar a los investigadores responsables de cada uno de ellos. Por su parte, sobre un total de cinco solicitudes de entrevistas realizadas a través del envío de correos y la realización de llamadas, se pudo concretar un total de tres conversaciones. ${ }^{2}$ Los ejes contemplados por estas fueron: período de trabajo, proceso de selección, temática abordada, actividades realizadas y resultados alcanzados, vínculos previos y posteriores a la iniciativa del Centro, aportes de las contrapartes al desarrollo del proyecto y balance de la experiencia. Con el objetivo de facilitar el análisis, las conversaciones fueron grabadas y transcritas en su totalidad. A continuación, se exponen los resultados y el análisis realizado sobre las fuentes mencionadas.

1 Los mismos cubrieron la totalidad de comunicados de prensa referidos a la cooperación bilateral con Cuba: "Barañao recibió a autoridades de Cuba", del 22 de junio de 2015; "El Ministro de Ciencia recibió al embajador de Cuba", del 9 de abril de 2014; "Barañao visita Cuba para fortalecer investigaciones conjuntas en biomedicina", del 18 de febrero de 2013.

2 Las entrevistas realizadas permitieron alcanzar la saturación de las categorías teóricas propuestas. Tanto al solicitar las entrevistas como al desarrollarlas, se explicó a cada entrevistado los objetivos y características de la investigación. Además, se solicitó el consentimiento para la grabación de la conversación y la reproducción de fragmentos, obteniendo una respuesta positiva de todos ellos. 


\section{Una mirada al marco formal de creación del CACBVaF}

Uno de los instrumentos para inducir, fomentar y orientar la cooperación internacional, son los acuerdos intergubernamentales e interinstitucionales, los cuales brindan el marco formal para las relaciones bilaterales y multilaterales (Sebastián, 2007). En el caso estudiado, se contó con el "Acuerdo Marco para la creación del Centro Binacional Argentino-Cubano de Biotecnología aplicada al Desarrollo de Vacunas y Fármacos”, suscrito por el MINCYT ${ }^{3}$ de la República Argentina, el Instituto Finlay (en adelante IF) y el Centro de Ingeniería Genética y Biotecnología (en adelante CIGB) de Cuba, en la ciudad de La Habana, el 19 de enero de 2009. El Acuerdo Marco examinado resalta la convicción de las partes sobre la importancia estratégica de la investigación científico-tecnológica en el área de la biotecnología aplicada al desarrollo de fármacos y vacunas, y la necesidad de perfeccionamiento de los recursos humanos y científicos de ambos países en dicho sector. Por su parte, los objetivos declarados en el documento son promover el intercambio y la transferencia de conocimientos científicos y tecnológicos, así como la formación y capacitación de recursos humanos en ambos países. Además, se propone elaborar y ejecutar proyectos orientados a la generación de conocimientos, productos y procesos en los temas de competencia del Centro.

Entre las modalidades de cooperación previstas en el Acuerdo Marco, se encuentran: el desarrollo conjunto y coordinado de proyectos de investigación científico-tecnológica; el intercambio y formación de expertos, científicos y técnicos; la organización de conferencias, seminarios, cursos de formación, especialización, perfeccionamiento profesional y adiestramiento; la utilización de equipos e instalaciones para el desarrollo conjunto de proyectos; y el intercambio de información científica y tecnológica, así como de políticas y experiencias de gestión en ese ámbito.

En lo concerniente a la organización institucional del Centro, el documento indica la existencia de una Dirección ejercida por un representante designado por cada una de las partes, la cual se ocupa de definir los programas de trabajo. Dicha Dirección se encuentra asesorada por un Comité Técnico integrado por dos representantes de la parte argentina y dos representantes de la parte cubana. El Comité asesora en la elaboración de los programas de trabajo y se encarga de examinar las posibilidades de cooperación en temas considerados estratégicos por las partes, con vistas a identificar y definir actividades conjuntas de investigación y desarrollo en el área de vacunas y fármacos. Los programas de trabajo identifican, además, las fuentes y mecanismos de financiamiento disponibles para apoyar su implementación. Posteriormente, son sometidos a consideración del MINCyT, del IF y del CIGB (Acuerdo Marco CACBVaF, 2009).

3 Cabe destacar que, a partir de 2018, el MINCYT fue relevado al rango de Secretaría de Ciencia, Tecnología e Innovación Productiva, dependiente del Ministerio de Educación, Cultura, Ciencia y Tecnología (Decreto Presidencial 802/2018).

144 Si Somos Americanos. Revista de Estudios Transfronterizos 
En cuanto al financiamiento, las partes se comprometen a proveer de forma equitativa los recursos necesarios para la ejecución de los programas de trabajo del CACBVaF. Además, en el documento de creación del Centro se prevé la firma de un Acuerdo de Confidencialidad con el objetivo de proteger la información, conocimiento (know how) y derechos de propiedad intelectual que se involucren en el desarrollo conjunto de los proyectos. Por otra parte, se estableció que todo conocimiento, derechos de propiedad intelectual o cualquier otro derecho que surja derivado del desarrollo conjunto, constituyen propiedad de las partes en las proporciones en que las mismas contribuyan. Finalmente, el Acuerdo quedó abierto a la participación de otros centros e instituciones.

Con base en lo examinado hasta aquí, puede decirse que el acuerdo expresa objetivos de cooperación convenidos de manera conjunta, referidos a la necesidad de perfeccionamiento de los recursos humanos, intercambio de información y producción de conocimientos, productos y procesos, en el marco de la importancia estratégica atribuida por las contrapartes a la biotecnología aplicada a la salud. Se proponen actividades concretas de cooperación, así como una dirección, asesoramiento y financiamiento compartidos, abonando la idea de compromiso mutuo y complementariedad. Asimismo, respecto de la cuestión de la simetría en la percepción de los beneficios, se propone, específicamente, contemplar los derechos de propiedad intelectual (u otros derechos) en relación con la contribución realizada por las partes.

Según la información brindada por la página web del MINCYT, el Centro contó con un total de cinco proyectos seleccionados, financiados y ejecutados, tres de los cuales se insertan en el área de Vacunas y Coadyuvantes y dos en el de Terapias contra el cáncer. A continuación se expone el análisis de las entrevistas a los directores de proyecto, contando con exponentes de ambas áreas. Cabe señalar que trascender el nivel declarativo de los acuerdos, depende de la concreción de los objetivos, así como del compromiso financiero y organizativo de los países suscriptores, siendo la ejecución de proyectos conjuntos de investigación, una de las actividades más genuinas de la cooperación científico-tecnológica internacional (Sebastián, 2004).

\section{Una mirada a las dinámicas de cooperación entre investigadores argentinos y cubanos en el marco del CACBVaF}

\section{Sobre la puesta en marcha del Centro y la selección de proyectos}

De acuerdo con la reconstrucción realizada a partir de las entrevistas, el período de trabajo en proyectos conjuntos de investigación en el marco del CACBVaF comenzó aproximadamente tres años después de la creación formal del Centro y se extendió hasta 2015. El proceso de selección se inició con la presentación de proyectos de cooperación 
entre grupos de investigación de Argentina y Cuba, en el marco de una convocatoria abierta realizada por el Centro. En consonancia con el Acuerdo Marco, dichas iniciativas fueron evaluadas por el ministerio argentino y por la contraparte cubana, quienes hicieron su respectiva ponderación y selección de aquellas propuestas de mayor interés para el país. La presentación y selección de proyectos de colaboración estuvo acompañada por la realización de encuentros entre los investigadores argentinos y cubanos en la ciudad de La Habana. Al respecto, dos de los entrevistados comentan que:

Diferentes grupos argentinos fueron convocados por el MINCYT para viajar a Cuba con el objetivo de estrechar lazos de colaboración. Se realizó un meeting entre grupos de investigación cubanos y argentinos para establecer los puntos de contacto. A partir de allí se presentaron proyectos a ser subsidiados y se seleccionaron los mejores proyectos de colaboración. (Entrevista a director de Proyecto B, 2017)

Se hizo un viaje con autoridades del ministerio para Cuba y se hicieron reuniones entre los diferentes centros de investigación y transferencia (...) Creo que fue un sensor para ver qué grado de interacción real existía entre lo que visualizaba el ministerio como posibilidad de interacción y lo que realmente había para debatir entre los institutos y los investigadores. Había proyectos que ya venían caminando (...) Había cosas que ya caían de maduro que podían tener una muy buena interacción. (Entrevista a director de Proyecto C, 2017)

En este punto, cabe señalar que la presencia de reconocimiento y confianza son características centrales de las colaboraciones científicas exitosas (Sebastián y Benavides, 2007), garantizando cierta viabilidad a los proyectos financiados con recursos públicos. En consonancia con esto, los centros binacionales se caracterizaron por articular polos existentes encargados de generar y difundir conocimientos en las áreas contempladas, identificados en las reuniones iniciales entre autoridades e investigadores. Las interacciones personales resultan importantes para dar un comienzo informal a la relación, mientras que la presencia de mecanismos estables de colaboración ofrece apoyo al trabajo colectivo y permite producir resultados diversos (D’Onofrio, Barrere, Fernández Esquinas y De Filippo, 2010). Si bien los acuerdos institucionales y gubernamentales pueden favorecer las relaciones entre los pares, carecen de piso sin la existencia previa de contactos concretos entre individuos (De Greiff, 1993).

Sobre las temáticas trabajadas y las fases de la investigación desarrolladas

En lo concerniente a las temáticas trabajadas en el marco del Centro, uno de los directores afirma haberse abocado al desarrollo de una vacuna acelular contra la tos convulsa, enfermedad infecto-contagiosa que aqueja mayormente a los niños de pocos meses y recién nacidos. El director del Proyecto comenta que: 
Tradicionalmente, esta enfermedad ha sido controlada a través de vacunas (...) realizadas a partir de células enteras de bacterias inactivas [que] traían consigo efectos secundarios (...) Se ha pasado a la elaboración de vacunas acelulares (...) Si bien estas nuevas vacunas redujeron las reacciones secundarias, la protección que otorgan es menor que la proporcionada por las vacunas a células enteras. Además, las bacterias evolucionan y las vacunas pierden eficiencia. Así, se buscan permanentemente nuevas estrategias, nuevos componentes, para formular nuevas vacunas. (Entrevista a director de Proyecto A, 2017)

De acuerdo con el entrevistado, el grupo de investigación al que pertenece es uno de los primeros que, a nivel internacional, propuso elaborar vacunas desde organismos en biofilm, reemplazando los reactores agitados mecánicamente. En este punto cabe señalar que, de acuerdo con Bourdieu (2000), el reconocimiento de los investigadores se relaciona con el valor distintivo y original realizado al cúmulo de conocimientos existente. Es por ello que, ser el primero - $\mathrm{O}$ estar entre los primeros- en hacer conocer y reconocer un determinado aporte, brinda un capital de autoridad importante. Con el paso del tiempo, el Laboratorio participó de proyectos de cooperación con Estados Unidos y Europa. Aquí se identifica la presencia de cooperación con actores científico-tecnológicos extrarregionales, lo cual permite acceder a importantes recursos económicos y simbólicos, así como generar productos que aumentan el prestigio y la visibilidad de los investigadores a nivel internacional, aunque muchas veces no se dan procesos de apropiación local (Kreimer, 2006).

En este caso, se buscaron socios del sector privado para continuar con el desarrollo de la vacuna. Cabe señalar que los gastos asociados con el desarrollo de vacunas y los derechos de propiedad intelectual que las protegen son cuantiosos, por lo que pocos laboratorios poseen la capacidad técnica para desarrollar completamente los productos (Homma, Di Fabio y De Quadros, 1998).

El desarrollo de vacunas se trata de un proceso complejo, costoso y largo, que involucra diversos actores como agencias de gobierno, institutos de investigación y desarrollo y empresas manufactureras, las cuales deben sortear un alto umbral de acceso (Corvalán, 2010).

En otro de los casos, el director de proyecto afirma haberse centrado en la evaluación de la combinación de la inmunoterapia con quimioterapia para el tratamiento de cáncer. En palabras del especialista:

Existen diferentes alternativas terapéuticas. Una de ellas es la vacuna, la cual prepara al sistema inmunológico para atacar a la célula tumoral durante la portación del cáncer. La quimioterapia, por su parte, es inmunosupresora, afecta al sistema inmunológico. El objetivo del proyecto fue encontrar la combinación con ambos tratamientos que mostrase el mayor beneficio. Se utilizaron modelos animales. (Entrevista a director de Proyecto B, 2017) 
En este caso, se avanzó sobre la etapa preclínica, incluyendo pruebas en animales. Las fases siguientes abarcan tratamientos clínicos en humanos y vigilancia postaprobación del ente regulador correspondiente (Corvalán, 2010). Otro de los directores comenta que su proyecto se centró en el desarrollo de una molécula peptídica que se aplica en tumores de cuello de útero y lesiones inducidas por Virus del Papiloma Humano (HPV). Al respecto, menciona que:

Ese financiamiento [obtenido en el marco del CACBVaF] se dedicó a una droga que tiene chances. El proyecto pasó por varias etapas, y llegó a la fase clínica, lo cual no quiere decir que esté en el mercado, porque son tres fases clínicas y la liberación (...) Cuando una droga tiene que avanzar a las fases clínicas, ya salís con millones de dólares (...) precisas un socio que en general es (...) una empresa privada. (Entrevista a director de Proyecto C, 2017)

El tratamiento clínico en humanos cubre tres fases, en las que se incrementa el número de voluntarios con quienes se testea el producto, estudiando distintos aspectos de eficacia y seguridad (Corvalán, 2010). De esta manera, los distintos proyectos han trabajado sobre temáticas pertinentes y relevantes para el Centro, que han permitido avanzar en etapas preclínicas y clínicas del desarrollo de vacunas, drogas y terapias para el tratamiento de diferentes enfermedades. Estos desarrollos han resultado, de acuerdo con los testimonios recabados, interesantes para científicos y empresas del ámbito internacional y nacional.

\section{Sobre las actividades realizadas y los lazos preexistentes}

En cuanto a las actividades y resultados, los directores de proyecto coinciden en señalar la realización de movilidades cubanas a la Argentina y movilidades en sentido contrario (las cuales varían de uno a tres casos por país), en las cuales se realizó entrenamiento, perfeccionamiento y difusión de resultados en congresos. Otras actividades llevadas a cabo en el marco de los proyectos refieren al dictado de cursos, la articulación académica entre las universidades implicadas para intercambios sobre la cuestión curricular y las publicaciones en coautoría internacional en revistas de corriente principal.

Tal como se mencionó al inicio, los entrevistados señalaron que las relaciones con las respectivas contrapartes cubanas databan de tiempo atrás. Los vínculos se iniciaron y sostuvieron en el tiempo a través de distintas actividades: participación en un curso dictado por el grupo argentino en el marco del Centro Argentino-Brasileño de Biotecnología (CABBIO), el cual contó con la participación de un investigador cubano; participación de un investigador argentino en un congreso en Cuba e intercambio posterior con director cubano; realización de pasantías por parte de recursos humanos cubanos en laboratorio argentino; participación conjunta en proyecto de investigación básica y trasnacional financiado por agencia argentina de promoción científico-tecnológica; participación conjunta en consorcios de composición público-privado para el desarrollo de productos tecnológicos asociados a la salud; trabajo colaborativo en el marco de proyectos de 
transferencia con empresas o subsidios nacionales e internacionales. En palabras de uno de los entrevistados:

El Centro Binacional es la entidad que soportó financieramente un proyecto de tres años, pero con el Centro de Ingeniería Genética y Biotecnología de la Habana venimos desarrollando una droga que pasó todas las instancias preclínicas, hasta pacientes, desde 2004. (Entrevista a director de Proyecto C, 2017)

En este punto es preciso señalar que los investigadores se mueven a través de redes bastante personales forjadas en seminarios, congresos y reuniones académicas, y puestas en marcha con el tiempo, a partir de las afinidades intelectuales y el interés por determinados temas (Meyer, Charum y Kaplan, 2001). Además, así como muchos lazos entre investigadores latinoamericanos surgen de la colaboración en programas extrarregionales, aquí se observa el caso de vínculos generados a partir de la participación en un centro argentino-brasileño de biotecnología.

De acuerdo con las entrevistas realizadas, la preexistencia de vínculos entre las contrapartes postuladas para el desarrollo de proyectos fue un elemento central contemplado en la selección de iniciativas. Ahora bien, el Centro les permitió obtener recursos para, en palabras de uno de los entrevistados, "sostener económicamente la relación". Al finalizar el financiamiento de esta iniciativa gubernamental, los lazos se volvieron más esporádicos, se desaceleró el ritmo de experimentación, se acudió a medios virtuales y telefónicos para sostener el contacto, se trabajó sobre la publicación de resultados y se recurrió a los recursos disponibles en otros proyectos en marcha, en el marco de los grupos de investigación involucrados. En este punto, cabe señalar que contar con fuentes de financiamiento nacionales genera un mayor grado de estabilidad y capacidad de negociación en la cooperación internacional (Sebastián, 2007).

\section{Sobre los aportes de las contrapartes y el trabajo conjunto}

Respecto de los aportes de las contrapartes al desarrollo del proyecto, los entrevistados coinciden en señalar la importante infraestructura y calidad científica con que cuenta la contraparte cubana para el desarrollo de las investigaciones. Cabe destacar aquí que un elemento central para la puesta en marcha de la colaboración científico-tecnológica refiere a la complementariedad de las capacidades, de manera de obtener en la vinculación resultados que no serían posibles de manera individual (Sebastián y Benavides, 2007). En el sector científico-tecnológico, los actores involucrados deben percibir la existencia de un beneficio para mantener el interés por la colaboración y considerar a la contraparte como un socio atractivo para las relaciones de trabajo científico (Kyvik, 2012).

Autores como Hubert y Spivak L'Hoste (2009) advierten que la carencia de los instrumentos requeridos para la producción de conocimiento es usualmente compensada por desplazamientos (nacionales e internacionales) hacia laboratorios equipados, así como 
por el trabajo conjunto en proyectos de colaboración internacional. Al respecto, uno de los testimonios advierte que:

En la investigación desarrollada [por mi grupo] se requiere contar con equipos de fermentación, reactores de mediana y gran escala (...) Este tipo de equipamiento y la infraestructura asociada al mismo se encuentra disponible en La Habana (...) Los cubanos habían publicado sobre formulaciones actuales de la vacuna acelular y tenían formas de producir estas proteínas en reactores a una escala mayor que el laboratorio argentino a través de organismos recombinantes. (Entrevista a director de Proyecto A, 2017)

Por su parte, los testimonios a continuación advierten sobre la combinación de distintas capacidades existentes en cada una de las contrapartes para el desarrollo de un "trabajo sinérgico". De acuerdo con los recursos disponibles en cada país, se da una "división del trabajo":

Fue un trabajo sinérgico, de crecimiento en un proyecto que apunta a mejorar la salud de la población. Cada grupo se aboca a un aspecto de la investigación: el grupo cubano a la producción de anticuerpos en grandes escalas y la evaluación de antígenos; el grupo argentino se abocó a modelos preclínicos. (Entrevista a director de Proyecto B, 2017)

El solape fue bárbaro porque ellos venían con un fuerte conocimiento en diseño de moléculas y síntesis (...) Y nosotros aportamos todo lo que es evaluación preclínica y diseño molecular de marcadores y demás. Y después en forma conjunta se hizo el diseño clínico de evaluación (...) Solapamos tecnología, combinamos lo que ellos tenían y nosotros no y viceversa. Fuimos a aprender cosas allá y ellos acá. (Entrevista a director de Proyecto C, 2017)

Esto permite relativizar la idea frecuentemente presente entre los investigadores latinoamericanos acerca de que tienen pocos recursos intelectuales, materiales y financieros para ofrecer a sus colegas (Velho, 2000), lo cual varía de acuerdo con los esfuerzos realizados por la política científico-tecnológica. En este punto, es preciso destacar una vez más la importancia de la reciprocidad en materia de cooperación científico-tecnológica, requiriendo de la consolidación de una comunidad científica nacional capaz de insertarse y proyectarse a nivel internacional (De Greiff, 1993). De una parte, los investigadores tienen que ser percibidos como socios "atractivos" para las relaciones de trabajo entre los pares del ámbito internacional (Kyvik, 2012). De otra, la consolidación de capacidades tiene que apuntar a la apropiación de los resultados de la cooperación, tendiendo a solucionar problemas del ámbito local (De Greiff, 1993).

\section{Sobre los aspectos positivos y negativos de la experiencia}

Finalmente, respecto del balance de la experiencia, se reconocen aspectos positivos como el financiamiento brindado por el Centro, el cual fue de 150.000 pesos anuales por un lapso de 
tres años (quedando pendiente el cobro de la última cuota al momento de la realización de las entrevistas). Uno de los entrevistados destaca que el dinero recibido en el marco del Centro posibilitó la compra de insumos, "tanto de material para cultivo de tejidos, como ensayos con animales" (Entrevista a director de Proyecto C, 2017). Asimismo, se afirma que "sin financiamiento no se puede hacer ciencia ni intercambio científico" (Entrevista a director de Proyecto B, 2017) ya que tanto los viajes como los insumos se pagan en moneda extranjera, con lo cual se requiere de montos importantes de financiamiento para cubrir los gastos de la investigación y el intercambio científico. En este punto, cabe señalar que los viajes y reuniones "cara a cara" resultan centrales en la vida de los científicos.

Otro de los entrevistados relativizó la importancia del financiamiento otorgado, en relación con los costos necesarios para atravesar la fase clínica y llegar al lanzamiento del producto al mercado, aunque considera que fue "una buena y novedosa iniciativa de la cual se extrae un balance positivo" (director de Proyecto B, 2017). En otro de los testimonios, se afirma que la experiencia promovió vínculos con contrapartes que "hablan de igual a igual" y donde los beneficios se distribuyen "50 y 50" (director de Proyecto C, 2017):

La iniciativa de fortalecer lo que venía en marcha fue muy buena (...) Ojalá existan muchas de estas iniciativas con países no solo del norte, sino con países como Cuba, que (...) tienen muy buena ciencia. El tema es que cuando firmás convenio con cualquier universidad de Estados Unidos, generalmente va todo a favor de ellos (...) todo el conocimiento queda en ellos. Acá queda 50 y 50. (Entrevista a director de Proyecto C, 2017)

Es difícil acceder a financiamiento europeo... hay mucho, pero para que, por ejemplo, Alemania coopere con Francia. Pero es difícil acceder desde América Latina. Hay pocos instrumentos de financiamiento de cooperación en Argentina y los de afuera no abundan y son difíciles de acceder. (Entrevista a director de Proyecto B, 2017)

El trabajo de De Filippo, Barrere y Gómez (2010) indica la existencia de dos tipos de cooperación internacional: la 'simétrica', en la que participan contrapartes con un grado de desarrollo científico y técnico similar y en la cual la aportación de cada investigador o institución es parecida; y la 'asimétrica', en la que participan contrapartes con un grado diferente de desarrollo científico y técnico, donde las aportaciones de cada uno de ellos son diferentes, así como la distribución de los beneficios. La asociación de contrapartes atravesadas por semejantes condiciones de desarrollo, desafíos y experiencias (como es el caso de los países de América Latina) permite la construcción de vínculos científicotecnológicos de carácter más horizontal y respetuosos de los intereses particulares de los cooperantes. Ahora bien, la disponibilidad de recursos financieros para sostener los vínculos entre países de menor desarrollo puede ser inestable.

Desde un punto de vista histórico, en contextos caracterizados por débiles sistemas científico-tecnológicos y escasas iniciativas gubernamentales para la promoción de la 
cooperación latinoamericana, las colaboraciones en materia científico-tecnológica entre los países de América Latina han tenido más bien un carácter informal y bilateral, asentándose en la proximidad geográfica y el conocimiento mutuo (Marí, Estébanez y Suárez, 2001). Los ejemplos de colaboración promovidos oficialmente refieren a experiencias puntuales, entre las que destaca el CABBIO, desarrollado desde la década de 1980 entre Argentina y Brasil (Hurtado, 2010). Por su parte, a principios del siglo XXI se reconocen dos procesos paralelos e interconectados: de una parte, la promoción de los sistemas científicotecnológicos latinoamericanos; de otra, el incremento de iniciativas y financiamiento para poner en marcha proyectos de cooperación entre contrapartes de América Latina (Zurbriggen y González Lago, 2010).

Entre los aspectos negativos, los testimonios señalan los retrasos en el pago de la última cuota del subsidio, la pérdida del poder de compra en dólares del monto total a lo largo de los años, la falta de financiamiento similar proveniente desde la contraparte cubana y la ausencia de nuevas convocatorias a proyectos conjuntos en el marco del Centro. ${ }^{4} \mathrm{Al}$ respecto, uno de los entrevistados advierte:

En la investigación (...) se requieren insumos para los trabajos experimentales -material biológico, reactores, organismos con patogenicidad -a una escala importante. En esta cuestión, la contraparte cubana disponía de menores posibilidades económicas que la Argentina para llevar a cabo trabajos experimentales, con lo cual se presentaron algunas limitaciones para llevar a cabo estudios de escalamiento de procesos. (Entrevista a director de Proyecto CACBVaF, 2017)

En este punto es preciso señalar la heterogeneidad existente al interior de los propios países de América Latina en su desarrollo, y la diferencia con países desarrollados en lo que concierne a disponibilidad de recursos. Por ejemplo, uno de los entrevistados afirmó que los pasantes argentinos en laboratorios de Alemania pudieron incluso extender sus estadías con fondos alemanes, "ya que la contraparte se interesa por el trabajo realizado y quiere que se llegue hasta las instancias finales" (Entrevista a director de Proyecto A, 2017). Los países desarrollados han implementado históricamente políticas deliberadas de atracción de científicos del ámbito internacional con el objetivo de aportar a sus procesos de innovación (Didou Aupetit, 2010).

4 Esta situación puede vincularse a la introducción de prioridades diferentes de política científico-tecnológica y política exterior por parte de la nueva administración a cargo del gobierno nacional desde fines de 2015. Esta hipótesis es solo tentativa y merecería el desarrollo de una nueva investigación centrada en un recorte temporal más reciente. 


\section{Una mirada a las relaciones entre Argentina y Cuba en el marco de la política exterior}

Respecto del análisis de la política exterior de Argentina hacia Cuba, se diferencian dos momentos: de una parte, lo ocurrido durante fines del siglo XX -signado por las presidencias de Carlos Menem (1989-1999) y Fernando De La Rúa (1999-2001)- y, de otra, lo sucedido a principios del siglo XXI -a partir del año 2002 con la asunción de Eduardo Duhalde a la presidencia argentina y, posteriormente, de Néstor Kirchner (20032007) y su sucesora Cristina Fernández de Kirchner (2007-2011; 2011-2015)- (Colombo y Giglio, 2011). A su vez, cabe tener presente que, de acuerdo con Simonoff (2009), las políticas exteriores argentinas se construyeron, desde 1980, a partir de un juego de equilibrios entre las tendencias autonomistas -que privilegian a la región como escenario principal de la agenda- y las de inserción con la potencia hegemónica -Estados Unidos.

De acuerdo con Colombo y Giglio (2011), entre 1989 y 2001 las relaciones con Cuba estuvieron condicionadas por el vínculo con los Estados Unidos, ya que la dirigencia argentina buscó forjar una relación especial con la potencia, esperando contar con su apoyo en temas estratégicos y sostener así el modelo neoliberal. En este marco, los gobiernos argentinos se alinearon con Washington en la posición adoptada sobre Cuba, manteniendo una retórica crítica respecto del régimen de Fidel Castro. Esta actitud generó tensión con el gobierno cubano, lo cual derivó en una confrontación directa y el retiro del embajador argentino de La Habana en 2001.

Ahora bien, a principios del siglo XXI, los nuevos gobiernos de la región latinoamericana se caracterizaron por el rechazo al neoliberalismo y un creciente sentimiento antiestadounidense. Las administraciones de carácter progresista coincidieron en la construcción de posiciones autónomas en el escenario internacional y en el avance en procesos de cooperación política y económica regionales, en los que consideraron también a Cuba (Colombo y Giglio, 2011). En el caso de Argentina, la opción regional fue uno de los contrapuntos con el discurso de los noventa que sobrestimó a los Estados Unidos como eje de relacionamiento externo: la política regional fue el área prioritaria, delimitada al plano sudamericano más que al latinoamericano, con la excepción de Cuba y México (Simonoff, 2009).

En abril de 2003, durante la 59 $9^{\circ}$ Reunión de la Comisión de Derechos Humanos de Naciones Unidas en Ginebra, el gobierno de Duhalde abandonó el voto de condena al régimen de Fidel Castro y optó por la abstención, contradiciendo la pretensión estadounidense. Dicha abstención marcó un cambio con respecto a la posición mantenida por Argentina hacia Cuba desde inicios de la década de los noventa, aunque el país continuó sin embajador en La Habana. A partir de 2003 hubo un mayor dinamismo en la relación. Ese año, Fidel Castro asistió a la ceremonia de asunción de la Presidencia de la Nación de Néstor Kirchner, siendo la primera vez que el dirigente cubano presenció la 
asunción de un presidente argentino y la tercera ocasión en que visitó el país. En octubre de ese mismo año, el canciller Bielsa encabezó una visita oficial a Cuba, con motivo de demostrar las intenciones del gobierno de estrechar relaciones y de presentar al nuevo embajador argentino en Cuba, Raúl Taleb. En esa ocasión, el canciller argentino y su par cubano Pérez Roque firmaron una serie de acuerdos de cooperación diplomática, cultural y comercial, y comenzaron negociaciones sobre el pago de la deuda que Cuba mantenía con Argentina desde 1974 (Aranda, 2004).

Ya en febrero de 2004, el canciller cubano realizó una visita oficial a Buenos Aires, donde fue recibido por el presidente Kirchner, declarando que los conflictos del pasado estaban superados y que el vínculo cubano-argentino se encontraba en un momento excelente. Durante las sucesivas cesiones de la Comisión de Derechos Humanos de Naciones Unidas, el gobierno argentino continuó optando por la abstención. En julio de 2006, con motivo de la reunión cumbre del MERCOSUR en Córdoba, Fidel Castro visitó nuevamente la Argentina, donde se acordó la firma de un Acuerdo de Complementación Económica con Cuba. $^{5}$

Cabe destacar que el documento "Lineamientos para la cooperación sur-sur (2013-2015)", emanado por el Ministerio de Relaciones Exteriores y Culto de la República Argentina, realiza una mención especial a la política de cooperación con el Caribe. Al respecto, se considera que el acercamiento a la región comenzó a principios del siglo XXI, cuando Argentina -según se consigna en el documento antes citado- "tomó la decisión estratégica de profundizar sus vínculos con los países del Caribe, incrementando el diálogo político y la cooperación bilateral" (p. 20).

En el marco de la colaboración, se desarrollaron misiones técnicas de relevamiento de temáticas prioritarias de cooperación en cada país (entre las que se encuentran las políticas de salud), se incrementó la cantidad de proyectos, se firmaron nuevos acuerdos de cooperación, tanto a nivel bilateral como multilateral con los organismos y foros de integración existentes en el Caribe. A partir de estas medidas, se buscó intensificar y dotar de perdurabilidad el vínculo y diálogo interregional.

Como balance de la relación, en el documento "Lineamientos para la cooperación sur-sur (2013-2015)" se destaca que: "Los proyectos con Cuba y República Dominicana han contribuido a fortalecer la investigación académica y el intercambio, especialmente en los sectores de salud y agricultura" (p. 12). Por su parte, como líneas de acción se advierte que, sin abandonar América Latina como área estratégica, durante el bienio 2013-2015 se buscaría profundizar las acciones de cooperación con regiones puntuales entre las que se encuentra el Caribe (junto con África y Asia).

5 Cabe mencionar que, a pesar del acercamiento entre los gobiernos de Argentina y Cuba, se registraron inconvenientes en las relaciones bilaterales, como es el caso de Hilda Molina, una médica cubana a quien se le denegaba persistentemente el permiso de salida para visitar a su familia radicada en Argentina. 
En el caso de Cuba, cabe señalar además que la cooperación sur-sur en el área específica de la salud forma parte de la política exterior del país. En 1962, Fidel Castro declaró en el acto de inauguración del Instituto de Ciencias Básicas y Preclínicas "Victoria de Girón" la decisión del gobierno cubano de brindar ayuda en el campo de la salud. Si bien existen antecedentes de este tipo de cooperación, se considera la fecha de inicio de la Colaboración Médica Internacional cubana con brigadas permanentes, el 23 de mayo de 1963, cuando se envió la primera brigada a Argelia. Desde entonces, esta iniciativa se sostiene acordemente con la propia evolución del Sistema Nacional de Salud, así como del contexto nacional e internacional más amplio (Marimón Torres y Martínez Cruz, 2010).

De acuerdo con lo mencionado hasta aquí, el perfil latinoamericanista de la política exterior argentina, la focalización estratégica del gobierno argentino en la región caribeña, la reanudación y estrechamiento de los lazos con Cuba y el sostenimiento de la cooperación sur-sur en salud como parte de la política exterior cubana, brindaron un marco propicio para poner en marcha iniciativas formales de cooperación bilateral entre ambos países, como es el caso del CACBVaF.

\section{Una mirada a la promoción de la biotecnología en el marco de la política científico-tecnológica}

La política científico-tecnológica argentina ha atravesado por distintas etapas al compás de las diversas orientaciones políticas de las administraciones (Albornoz y Gordon, 2011). Entre 2003 y 2015, los gobiernos de Néstor Kirchner y Cristina Fernández de Kirchner posicionaron a la ciencia y la tecnología como un área central y una política permanente del Estado, generándose cambios importantes de carácter institucional, instrumental y presupuestario. Los principales ejes de la política de ciencia y tecnología del período han sido: incremento del presupuesto público destinado a la financiación de la ciencia y la tecnología, aumento del número de investigadores y becarios, recuperación de salarios y estipendios, incremento del financiamiento de proyectos en variadas modalidades, repatriación de investigadores argentinos en el exterior, elaboración de planes estratégicos en el área y la mejora y creación de infraestructura (Naidorf, Perrotta, Gómez y Riccono, 2015).

En el ámbito institucional, en 2007 se creó el MINCYT, lo cual implicó la presencia directa de la problemática científica y tecnológica en el proceso de toma de decisiones a nivel país. El ministerio, a través de la Dirección Nacional de Relaciones Internacionales, promovió el fortalecimiento de la cooperación internacional como herramienta estratégica para el desarrollo de la ciencia, la tecnología y la innovación productiva en el ámbito nacional, colocando al entorno más inmediato -el MERCOSUR- y a América Latina, en general, como socios prioritarios en la colaboración más allá de las fronteras (López, 2017). 
Es preciso destacar que la biotecnología fue uno de los sectores prioritarios para la política científico-tecnológica del período (Naidorf, Perrotta, Gómez y Riccono, 2015), reflejado en el gran crecimiento registrado tanto en el número de grupos abocados a actividades de investigación en biociencias y biotecnología, como los mayores presupuestos operativos y las inversiones en infraestructura (Anlló et al., 2016).

Ahora bien, las capacidades argentinas en biotecnología son el fruto de varias décadas de esfuerzos en investigación y desarrollo en materia de biología avanzada, biomedicina, ciencias agrarias, veterinarias y otras áreas relacionadas con recursos naturales. Argentina desarrolló tempranas capacidades científicas que fueron un excelente punto de partida de los desarrollos biotecnológicos recientes (Anlló et al., 2016). Hacia la década de 1980, la administración de Alfonsín colocó a la biotecnología y a la informática como las dos áreas más dinámicas del campo científico, generándose importantes y duraderas iniciativas de colaboración con Brasil en la materia, como el CABBIO (Hurtado, 2010).

En 1985 se realizó el Encuentro Argentino-Brasileño sobre Biotecnología, cuyo resultado fue la creación de un grupo de trabajo binacional encargado de la organización del CABBIO, al que luego se agregaron Chile, Paraguay y Uruguay (Mallo, 2011). El centro no se cristalizó en una estructura física centralizada, sino que funciona como una red de grupos de investigación y desarrollo en biotecnología de ambos países, cuyo objetivo es promover las interacciones entre actores públicos y privados mediante la implementación de proyectos binacionales, así como la capacitación de recursos humanos a través de la Escuela Argentina-Brasileña de Biotecnología (EABBIO) (Arza y Carattoli, 2012). Por entonces comenzaron a aparecer en el mercado los primeros productos biotecnológicos de producción nacional aplicados a la salud humana y a la genética vegetal, con lo que Argentina ingresó tempranamente al conjunto de países líderes (Anlló et al., 2016).

El Plan Nacional de Ciencia, Tecnología e Innovación "Argentina Innovadora 2020" incluyó a la biotecnología entre sus áreas prioritarias. Además, diversas instituciones gubernamentales pusieron en marcha programas de apoyo al sector biotecnológico, entre las que se destaca la Agencia Nacional de Promoción Científico-Tecnológica con sus instrumentos dirigidos a la cooperación público-privada para innovación en biotecnología. También resaltan los proyectos de cooperación internacional en biotecnología del MINCYT, los programas de apoyo a la formación de recursos humanos en el sistema científico y en las empresas, y el desarrollo de carreras de grado y posgrado en biotecnología en diversas universidades.

Hacia 2014, Argentina contó con recursos humanos calificados en ciencias básicas e ingeniería abocados a un amplio espectro de ramas de las biociencias y, en alguna medida, en biotecnologías; equipamiento básico disponible para investigaciones en los principales centros de investigación y desarrollo dedicados a la biotecnología; y una base empresaria consolidada, dedicada a la producción de bienes y servicios de biotecnología. En cuanto a las áreas de aplicación, el área de salud humana concentraba el 54\% de los esfuerzos, 
seguidos por aplicaciones en biotecnología agropecuaria (37\%) y en salud animal (35\%) (Anlló et al., 2016).

Entre las debilidades de la biotecnología argentina se destacan: la escasa orientación del sistema científico-tecnológico a la producción de innovaciones con uso comercial definido; el tamaño relativamente pequeño de los grupos dedicados a la investigación en biotecnología; la falta de equipamiento para las etapas de escalado; el tamaño pequeño de las firmas locales; y, a pesar de los avances en la materia, el limitado conocimiento por parte de las empresas de las potencialidades y áreas de especialización del sistema científico del sector (Anlló et al., 2016).

Cabe contemplar que entre 2013 y 2015 se sostuvieron reuniones oficiales entre autoridades del área científico-tecnológica de Cuba y Argentina, así como se produjo la visita de centros de investigación y referentes industriales en biotecnología. Entre los actores involucrados se destacan las autoridades del Ministerio de Ciencia, Tecnología e Innovación Productiva y el Ministerio de Relaciones Exteriores de Argentina y diversas contrapartes cubanas, como el Ministerio de Ciencia, Tecnología y Medio Ambiente, el Ministerio de Salud Pública, el Ministerio de Comercio Exterior y la Inversión Extranjera, el Ministerio de Relaciones Exteriores y la Embajada de Cuba en Argentina. También participaron centros de investigación y organizaciones industriales cubanas como el Centro de Ingeniería Genética y Biotecnológica, el Centro de Inmunología Molecular, BioCubaFarm y el Instituto Finlay.

De acuerdo con los comunicados de prensa analizados, en dichas reuniones se conversó sobre la importancia de fortalecer las relaciones bilaterales entre ese país y la Argentina en materia de ciencia y tecnología; la existencia de espacios para la complementariedad entre Argentina y Cuba de cara a la obtención de beneficios económicos y sociales para ambas partes; los avances científicos contra el cáncer; la presencia de la biotecnología como sector de trabajo prioritario, en el que ambos países presentan un significativo avance; y el desempeño y resultados destacables del CACBVaF.

El Acuerdo Marco resalta que el MINCyT ha planteado como objetivo de su gestión la solución de problemas estructurales y el desarrollo de proyectos científico-tecnológicos de alto impacto en el área de salud. ${ }^{6}$ Asimismo, destaca que los centros cubanos de investigación, desarrollo, producción y comercialización de vacunas y fármacos cuentan con importantes logros científicos en la materia y disponen de la contribución de más de 2.300 trabajadores de alta calificación y experiencia en el campo. Igualmente, se subrayan las "modernas instalaciones donde se investiga y produce dentro de los más exigentes requisitos establecidos por las entidades competentes" (Acuerdo Marco, 2009, p. 1).

6 En el caso de Argentina, el período estudiado se caracteriza por la recuperación de la presencia estatal en el sector, con medidas y programas de importancia relativos, por ejemplo, a la política de medicamentos (Alonso y Di Costa, 2015). 
En el caso de Cuba, las posibilidades de cooperar internacionalmente en biotecnología aplicada al desarrollo de vacunas y fármacos se derivan del importante desarrollo de su Sistema Nacional de Salud, asentado sobre el principio de la salud como un derecho social inalienable de toda la población y como una responsabilidad del Estado. Cuba cuenta con capital humano y capacidades nacionales fundamentales en el área de salud; ha invertido el porcentaje más alto del PIB en América Latina en el área, a la vez que la investigación resulta una actividad prioritaria en todas las instituciones de salud. En lo que refiere a la solidaridad internacional, la Cooperación Sur-Sur resulta fundamental para el gobierno cubano, especialmente en la transferencia de tecnologías y ayuda médica (DomínguezAlonso y Zacca, 2011; Marimón Torres y Martínez Cruz, 2011).

Si bien la voluntad del Estado cubano hacia el desarrollo de las ciencias estuvo desde los inicios del proceso revolucionario, desde mediados de los años ochenta el país introdujo la definición de prioridades nuevas para el desarrollo científico y tecnológico, como las biociencias, la biotecnología, la industria farmacéutica y los equipos médicos de alta tecnología (García Fernández y Chassagnes Izquierdo, 2003). En la década de 1990, la política científico-tecnológica cubana se caracterizó por la creación de un Sistema Nacional de Ciencia e Innovación Tecnológica, sustentado en la centralidad de la innovación, una renovada racionalidad tecnológica de evaluación de tecnologías y la consolidación de nexos entre la ciencia, la tecnología y la sociedad (Núñez Jover, 2005).

Desde la política científico-tecnológica cubana se han promovido históricamente cambios institucionales y se ha puesto en marcha una red de instituciones científicas que facilitaron el desarrollo de las biociencias y la biotecnología. De acuerdo con García Fernández y Chassagnes Izquierdo (2003), la biotecnología cubana comenzó como "adoptador temprano" de tecnologías desarrolladas por otros países. Ahora bien, la transferencia inicial de la tecnología extranjera generó un proceso interno de aprendizaje. Paulatinamente se mejoraron las tecnologías importadas, se desarrollaron capacidades innovativas propias y se crearon nuevos conocimientos cercanos a la frontera tecnológica.

A partir de 1980 surgieron varias instituciones científicas y productivas que asimilaron y desarrollaron rápidamente la biotecnología, conformando el Polo Científico de La Habana. Dicho complejo cuenta con más de 20 instituciones y más de 10.000 trabajadores (superando la cantidad de 4.000 científicos e ingenieros). Entre sus resultados, puede mencionarse que los productos allí creados han beneficiado a cientos de miles de cubanos y se han exportado a más de 50 países. Por ejemplo, Cuba ha aportado 16 productos terapéuticos principales para cáncer, contando además con más de 600 productos en ensayo clínico al año 2011 (Lage, 2011).

En términos generales, el desarrollo de la biotecnología cubana se sustenta en principios como: el Estado cubano como único inversionista; la biotecnología como parte del sistema de salud cubano priorizando sus necesidades; el desarrollo y capacitación de recursos humanos propios; el desarrollo de las operaciones a ciclo cerrado, desde la investigación 
hasta la comercialización, con una sostenibilidad a partir de las propias exportaciones del sector; la integración y cooperación entre las instituciones participantes, haciendo un uso racional de las potencialidades de cada una; desarrollo de nuevas instituciones y empresas derivadas de la salida de nuevos resultados y producciones; mejora constante de la calidad, desarrollo de las buenas prácticas y sistema de calidad, así como de la novedad e impacto de los productos (Herrera Martínez, 2011). Lage (2012) afirma que la biotecnología se ha convertido en un sector industrial, exportador e innovador que surge a partir de la inversión estatal.

Según Núñez Jover (2005), Cuba es un país cuyo desarrollo científico, medido según los indicadores internacionales, presenta una posición favorable dentro de los países de América Latina y el Caribe. Sobre todo en áreas como la biotecnología y la industria médico-farmacéutica, constituye una reconocida potencia científica. La capacidad técnica y la fortaleza institucional disponibles en Cuba permiten su participación en redes internacionales y el aprovechamiento de la cooperación internacional según criterios endógenos y los problemas relevantes del desarrollo local.

En el marco del reconocimiento del temprano y potente desarrollo cubano en biotecnología aplicada a la salud, así como de la potenciación de las capacidades científico-tecnológicas biotecnológicas argentinas -en términos de recursos humanos, financiamiento e infraestructura- durante el período estudiado, se encontraron condiciones propicias para la puesta en marcha de iniciativas formales, como es el caso del CACBVaF, y para la promoción del trabajo conjunto entre grupos e institutos de investigación argentinos y cubanos en la materia.

\section{Discusión de los resultados}

Al analizar conjuntamente los resultados obtenidos del examen de las diferentes dimensiones, puede plantearse que la política científico-tecnológica argentina, en consonancia con los lineamientos de política exterior del período, dotó de un paraguas formal (a partir de la firma del Acuerdo Marco) y de recursos económicos (a través del financiamiento de proyectos conjuntos de investigación), y fortaleció lazos de cooperación argentino-cubanos preexistentes, para un desarrollo más estable y dinámico de los mismos. Los lazos previos entre investigadores de ambos países resultaron una plataforma fundamental para garantizar la viabilidad de la iniciativa formal propuesta en el Acuerdo Marco. El éxito de la cooperación científico-tecnológica internacional depende, en gran parte, del reconocimiento y confianza existente entre los actores.

A su vez, el Acuerdo Marco estipuló las condiciones formales de la relación, señalando la importancia estratégica de la investigación científico-tecnológica en el sector, advirtiendo una dirección, asesoramiento y financiamiento compartido, previendo el beneficio mutuo 
en el intercambio de conocimientos y la formación de recursos humanos, considerando la realización de actividades concretas de cooperación, y proponiendo la distribución de la participación en los derechos de propiedad intelectual en función de los aportes realizados. En el marco del Centro constituido, se realizó una convocatoria para el financiamiento de proyectos conjuntos de investigación, evaluados y seleccionados de manera conjunta.

Se considera que la convocatoria, selección y financiamiento de proyectos en el marco del Centro, permitió dar estabilidad a los lazos preexistentes, en el sentido de que se pudieron planificar y concretizar viajes de intercambio, lo cual resulta importante para nutrir los vínculos, compartir conocimientos tácitos y utilizar equipamiento inexistente en el contexto local, más allá de la complementariedad ofrecida por las nuevas tecnologías de la información y la comunicación. A su vez, se dinamizaron los vínculos, en tanto se contribuyó al despliegue de distintas actividades, el avance sobre distintas etapas de la investigación y el relacionamiento con distintos actores involucrados en el desarrollo biotecnológico. Cabe señalar que el apoyo recibido desde el CACBVaF para el desarrollo de proyectos conjuntos, fue complementado a partir de otras políticas e instrumentos relacionados -mencionados por los entrevistados-, en un contexto donde la ciencia y la tecnología cobraron relevancia para la política pública.

La experiencia, aunque en términos generales fue caracterizada de manera positiva por los actores entrevistados, no estuvo exenta de limitaciones. En la comparación con actividades de cooperación científico-tecnológica con contrapartes de mayor desarrollo relativo, los entrevistados identificaron aspectos positivos, como la distribución más equitativa de los beneficios en la vinculación con colegas de la región, y aspectos negativos, como la menor disponibilidad de fondos para el sostén de lazos latinoamericanos. También destacaron la mayor competitividad existente entre los fondos de financiamiento de la cooperación internacional extrarregional, aunque señalaron que, en Argentina, los recursos económicos destinados a dicha actividad tampoco son abundantes.

Si bien se consideraron los aspectos positivos de contar con el financiamiento provisto por el CACBVaF, también se señalaron limitaciones, tanto en lo referido al nivel de los fondos, los cuales resultaron escasos frente a los costos de escalamiento y avance hacia etapas clínicas del proceso, como a su poder de compra en dólares, de acuerdo con la política macroeconómica argentina y su relación específica con la moneda extranjera. Asimismo, se advirtieron problemas en la recepción de los fondos, la ausencia de nuevas convocatorias a proyectos conjuntos de investigación en el marco del Centro y el nivel de aporte económico de la contraparte cubana. En fin, se llevaron adelante cinco proyectos, constituyéndose en una experiencia acotada, aunque cabe resaltarla como una expresión de un tipo de cooperación científico-tecnológica distinta a la promovida tradicionalmente con países del "Norte". 


\section{Reflexiones finales}

El presente trabajo se propuso contribuir al estudio de la cooperación científico-tecnológica entre los países de América Latina, a partir de examinar la cooperación entre Argentina y Cuba a principios del siglo XXI. Desde el punto de vista teórico, recuperó aportes de la sociología de la ciencia, las relaciones internacionales y la política científica. Desde el punto de vista metodológico, abordó el caso del Centro Argentino-Cubano de Biotecnología Aplicada al desarrollo de Vacunas y Fármacos, creado conjuntamente por el Ministerio de Ciencia, Tecnología e Innovación Productiva y contrapartes cubanas. El período temporal se extendió entre 2009 y 2015, desde la firma del Acuerdo Marco hasta la finalización de los proyectos seleccionados y desarrollados en su seno.

Se considera que la cooperación entre Argentina y Cuba en el marco del Centro se explica a partir del entrelazamiento de distintos factores. En el nivel microsocial se destaca la preexistencia de vínculos entre científicos argentinos y cubanos desarrollados con anterioridad a la constitución del Centro, basados en una percepción sobre los beneficios mutuos, la complementariedad de recursos, la simetría y el respeto. Desde el punto de vista de las relaciones internacionales, en el período estudiado se retomaron y profundizaron los lazos con Cuba, suspendidos hacia fines del siglo XX. Por su parte, la política científicotecnológica argentina apuntaló el desarrollo de la biotecnología, así como la cooperación bilateral con Cuba.

En investigaciones futuras cabría indagar en la cooperación con contrapartes cubanas en momentos más recientes para establecer líneas de comparación en torno a las dinámicas microsociales de la cooperación internacional, la incidencia de la política exterior y la influencia de la política científico-tecnológica, de modo de retomar los aportes de distintas disciplinas.

\section{Referencias}

Acuerdo Marco, MINCyT (19 de enero, 2009). Acuerdo Marco para la Creación del Centro Binacional Argentino-Cubano de Biotecnología Aplicada al Desarrollo de Vacunas y Fármacos-CACBVAF. La Habana, República de Cuba.

Albornoz, M. y Gordon, A. (2011). La política de ciencia y tecnología en Argentina desde la recuperación de la democracia (1983-2009). En M. Albornoz y J. Sebastián (Eds.), Trayectorias de las políticas científicas y universitarias de Argentina y España (pp. 1-46). Madrid: CSIC.

Alonso, G. A. y Di Costa, V. (2015). Más allá del principio contributivo: cambios y continuidades en la política social argentina, 2003-2011. Estudios Sociológicos, 13(9), 31-62. 
Anlló, G., Añon, M. C., Bassó, S., Bellinzoni, R., Bisang, R., Cardillo, S. Regunaga, M. (2016). Biotecnología argentina al año 2030: llave estratégica para un modelo de desarrollo tecno-productivo. Buenos Aires: MINCYT.

Aranda, R. A. (2004). La política exterior argentina: De Menem a Kirchner. Relaciones Internacionales, 27, 39-58.

Arza, V. y Carattoli, M. C. (2012). El desarrollo de la biotecnología y las vinculaciones público-privadas, una discusión de la literatura orientada al caso argentino. Realidad Económica, 266, 49-71.

Bourdieu, P. (2000). El campo científico. Los usos sociales de la ciencia (pp. 11-27). Buenos Aires: Ediciones Nueva Visión.

Colombo, S. y Giglio, A. (2011). La vinculación con Cuba: factores que explican una nueva política en el período pos neoliberal. En S. Colombo (Comp.), La inserción internacional de Argentina durante la presidencia de Néstor Kirchner: un cambio de época (pp. 74-102). Tandil: UNCPBA.

Corvalán, D. (2010). Estudios sobre los desarrollos internacionales de la biotecnología industrial: vacunas humanas. Documentos de Trabajo del CEUR-CONICET. Recuperado de http://www.ceurconicet.gov.ar/archivos/publicaciones/PICTDto8vacunas1.pdf

De Filippo, D., Barrere, R. y Gómez, I. (2010). Características e impacto de la producción científica en colaboración entre Argentina y España. Revista Iberoamericana de Ciencia, Tecnología y Sociedad, 6(16), 1-20.

De Greiff, A. (septiembre, 1993). Cooperación internacional en ciencia y tecnología: reflexiones en torno al caso colombiano. X Encuentro Nacional de Informática realizado en la Universidad Externado de Colombia, Colombia.

DGCIN (s/f). Lineamientos para la cooperación sur-sur (2013-2015). Dirección General de Cooperación Internacional, Argentina.

Díaz, C. y Navarro, P. (1998). Análisis de contenido. En J. M. Delgado y J. Gutiérrez (Coords.), Métodos y técnicas cualitativas de investigación en ciencias sociales (pp. 177-224). Madrid: Síntesis.

Didou Aupetit, S. (2010). De la fuga de cerebros a la atracción de conocimientos en las ciencias sociales de América Latina. En UNESCO (Ed.), Informe mundial sobre las ciencias sociales. Divisorias del conocimiento (pp. 125-127). París: Ediciones UNESCO.

Domínguez-Alonso, E. y Zacca, E. (2011). Sistema de salud de Cuba. Salud Pública de México, 53(2), 168-176.

D'Onofrio, M. G., Barrere, R., Fernández Esquinas, M. y De Filippo, D. (2010). Motivaciones y dinámica de la cooperación científica bilateral entre Argentina y España: la perspectiva de los investigadores. Revista Iberoamericana de Ciencia, Tecnología y Sociedad, 6(16), 213-236. 
García Fernández, F. y Chassagnes Izquierdo, O. (2003). Políticas de innovación en Cuba: una revisión de las políticas aplicadas en el desarrollo de la industria biotecnológica asociada a la salud. Revista Iberoamericana de Ciencia, Tecnología y Sociedad, 6, $1-15$.

Herrera Martínez, L. S. (2011). 25 años del Centro de Ingeniería Genética y Biotecnología. Revista Anales de la Academia de Ciencias de Cuba, 1(2), 1-12.

Herrera, A. (2015). Ciencia y política en América Latina. Buenos Aires: Biblioteca Nacional.

Homma, A., Di Fabio, J. L. y De Quadros, C. (1998). Los laboratorios públicos productores de vacunas: el nuevo paradigma. Revista Panamericana de Salud Pública, 4(4), 223-232.

Hubert, M. y Spivak L'Hoste, A. (2009). Integrarse en redes de cooperación en nanociencias y nanotecnologías: el rol de los dispositivos instrumentales. Redes, 15(29), 69-91.

Hurtado, D. (2010). La ciencia argentina: un proyecto inconcluso: 1930-2000. Buenos Aires: Edhasa.

Hurtado, D. (2012). La colaboración científica en dos ejes de cooperación clave: Sur-Sur (S-S) y Norte-Sur (N-S). En MINCYT (Ed.), Hacia un mejor aprovechamiento de la cooperación internacional para el fortalecimiento del Sistema Nacional de Ciencia, Tecnología e Innovación (pp. 24-27). Buenos Aires: MINCYT.

Kern, A. (1-2 de octubre, 2009). La cooperación científica y tecnológica como campo de estudio en las relaciones internacionales. Jornadas de Relaciones Internacionales, FLACSO-Argentina.

Kern, A. (23-25 de julio, 2014). La agenda científica y tecnológica en los regionalismos de América Latina. Conferencia Internacional Conjunta FLACSO-ISA, Buenos Aires, Argentina.

Kreimer, P. (1999). De probetas, computadoras y ratones. La construcción de una mirada sociológica sobre la ciencia. Bernal: Universidad Nacional de Quilmes.

Kreimer, P. (2006). ¿Dependientes o integrados? La ciencia latinoamericana y la división internacional del trabajo. Nómadas, 24, 199-212.

Kyvik, S. (2012). Trabajo en red, colaboración y publicaciones como medios de internacionalización de la investigación. En N. Fernández Lamarra y M. Marquina (Comps.), El futuro de la profesión académica. Desafíos para los países emergentes (pp. 318-328). Buenos Aires: EDUNTREF.

Lage, A. (2011). El espacio de la biotecnología en el control del cáncer: oportunidades y desafíos en Cuba. Revista Cubana de Salud Pública, 37, 661-674.

Lage, A. (2012). Las funciones de la ciencia en el modelo económico cubano: intuiciones a partir del crecimiento de la industria biotecnológica. Economía y Desarrollo, 147(1), 80-106. 
López Noguero, F. (2002). El análisis de contenido como método de investigación. Revista de Educación, 4, 167-179.

López, M. P. (2017). La cooperación en ciencia y tecnología entre Argentina y los países de América Latina. El caso del Ministerio de Ciencia, Tecnología e Innovación Productiva (2007-2015). Cuadernos de Política Exterior Argentina, 126, 31-46.

Mallo, E. (2011). Políticas de ciencia y tecnología en la Argentina: la diversificación de problemas globales, ¿soluciones locales? Redes, 17(32), 133-160.

Marí, M., Estébanez, M. E. y Suárez, D. (2001). La cooperación en ciencia y tecnología de Argentina con los países del Mercosur. Redes, 8(17), 59-82.

Marimón Torres, N. y Martínez Cruz, E. (2011). Experiencia cubana en Cooperación SurSur. Revista Cubana de Salud Pública, 37(4), 380-393.

Meyer, J. B., Charum, J. y Kaplan, J. (2001). El nomadismo científico y la nueva geopolítica del conocimiento. Revista Internacional de Ciencias Sociales, 168, 170185.

Naidorf, J., Perrotta, D., Gómez, S. y Riccono, G. (2015). Políticas universitarias y políticas científicas en Argentina pos 2000. Crisis, innovación y relevancia social. Revista Cubana de Educación Superior, 34(1), 10-28.

Núñez Jover, J. (2005). Notas sobre ciencia, tecnología y sociedad en Cuba. La Habana: Universidad de La Habana.

Russell, J., Ainsworth, S., Del Río, J., Narváez-Berthelemot, N. y Cortés, H. (2007). Colaboración científica entre países de la región latinoamericana. Revista Española de Documentación Científica, 30(2), 180-198.

Sebastián, J. (2004). Cooperación e internacionalización de las universidades. Buenos Aires: Biblos.

Sebastián, J. (2007). Conocimiento, cooperación y desarrollo. Revista Iberoamericana de Ciencia, Tecnología y Sociedad, 3(8), 195-208.

Sebastián, J. y Benavides, C. (2007). Ciencia, tecnología y desarrollo. Madrid: Agencia Española de Cooperación Internacional.

Simonoff, A. (2009). Regularidades de la política exterior de Néstor Kirchner. Confines, 5(10), 71-86.

Velho, L. (2000). Redes regionales de cooperación en CyT y el Mercosur. Redes, 7(15), 112-130.

Zurbriggen, C. y González Lago, M. (2010). Análisis de las iniciativas Mercosur para la promoción de la ciencia, la tecnología y la innovación. Montevideo: Centro de Formación para la Integración Regional. 